\title{
Bullous leukemia cutis mimicking facial cellulitis ${ }^{*}$
}

\author{
Luciana de Sales Caldato ${ }^{1}$ \\ Ligia Niero-Melo ${ }^{1}$
}

\author{
Juliana de Sousa Britto ${ }^{1}$ \\ Hélio Amante Miot ${ }^{1}$
}

Abstract: Bullous leukemia cutis is an uncommon clinical manifestation of cutaneous infiltration by leukemic cells, from B-cell chronic lymphocytic leukemia. We present the case of a 67-year-old, female, chronic lymphocytic leukemia patient. She was taking chlorambucil and developed facial edema with erythema and warmth, misjudged as facial cellulitis. Two days later, she developed bullous lesions in the arms, legs, neck and face. The histopathology of facial and bullous lesions confirmed leukemia cutis. All lesions disappeared following the administration of rituximab combined with cycles of fludarabine and cyclophosphamide. Although soft tissue infections are common complications in patients undergoing chemotherapy, leukemia cutis can also resemble cellulitis.

Keywords: Dermatology; Leukemia; Skin diseases, vesiculobullous

Leukemia cutis is a cutaneous infiltration by leukemic cells, occurring in $5-20 \%$ of all leukemia cases. Bullous leukemia cutis (BLC) is an uncommon clinical subtype, associated with B-cell chronic lymphocytic leukemia (CLL). ${ }^{1,2}$ Here, we describe a patient with CLL who developed BLC and facial lesions that mimicked cellulitis.

A 67-year-old woman with CLL who had been taking chlorambucil for the past year developed a periorbital and malar edema with erythema and warmth, despite having no fever or pain (Figure 1). She was admitted to the dermatology ward and administered amoxicillin-clavulanate. Biochemical exams were unremarkable and the hemogram showed leukocytosis $\left(24200 / \mathrm{mm}^{3}\right)$ with lymphocytosis $(73.3 \%)$. Forty-eight hours later, bullous lesions emerged on the arms, legs,

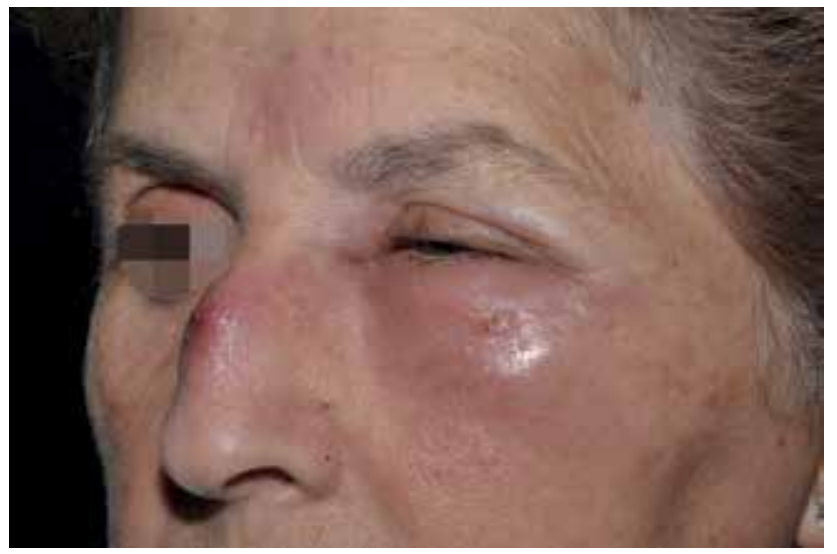

Figure 1: Bullous leukemia cutis mimicking facial cellulitis. Periorbital and malar erythema and edema the facial edema (Figure 2).

1 Universidade Estadual Paulista “Júlio de Mesquita Filho" (Unesp) - Botucatu (SP), Brazil. 


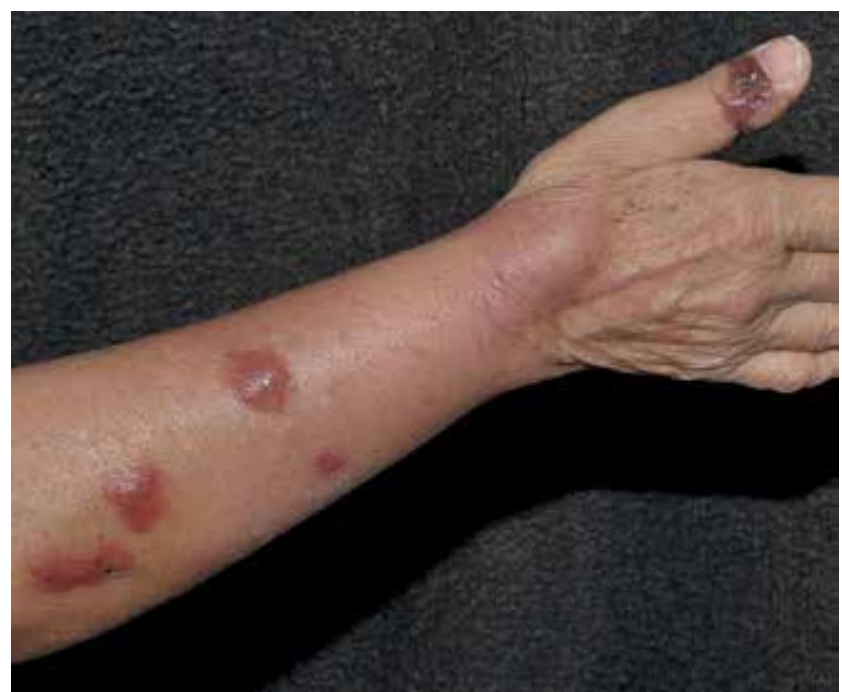

Figure 2: Bullous leukemia cutis. Multiple bullous lesions on the right forearm and thumb

Histopathology on facial and bullous lesions revealed dense, cutaneous infiltration by small, monomorphous, hyperchromatic lymphocytes. Further, the immunohistochemistry study was positive for CD20, CD5, CD 23, CD43 and ZAP-70.

The patient underwent a chemotherapic regimen with five cycles of cyclophosphamide, prednisone, vincristine and doxorubicin; four rituximab cycles were also administered. As no significant clinical improvement was noted, rituximab was combined with fludarabine and cyclophosphamide cycles, entailing gradual disappearance of cutaneous lesions despite the continued high bone marrow cellularity.

CLL is the most prevalent chronic leukemia in adulthood (3-5 cases $/ 100,000$ people) and $90 \%$ of cases occur after the age of $50 .{ }^{3}$ Cutaneous lesions in CLL can be specific or not. Sweet's syndrome, herpes zos- ter, erythema nodosum, skin infections (bacterial and fungal), drug reactions and insect bite reactions have been described. ${ }^{4}$

The hypothesized mechanism of cutaneous infiltration is the migration of lymphocytes from the vasculature, mediated by intercellular adhesion molecule-1 (ICAM-1) and lymphocyte function-associated antigen-1 (LFA-1). ${ }^{4}$

Depending on the pattern of cutaneous infiltration (epidermis, dermis or subcutaneous fat), leukemia cutis can be characterized by papules, plaques, patches, purpuric lesions, nodules, bullae or ulcers. It can arise at any stage of disease, although in 5-18\% of cases it can precede the diagnosis. Leukemia cutis can affect any cutaneous site but it manifests most often as papules and nodules on the face, chest and extremities. There is no difference in clinical patterns according to leukemia type, though erythrodermia and BLC are rare subtypes reported only in CLL; gingival hypertrophy, in acute myeloid leukemia, and vesicles, in acute granulocytic leukemia. ${ }^{1,5}$

Leukemia cutis is generally associated with a more aggressive disease and poor prognosis, except for CLL. ${ }^{1,4,6}$ Conversely, our patient has immunophenotype ZAP-70 and has experienced incomplete disease remission after different chemotherapy schemes, indicating a less responsive disease. ${ }^{3}$

Treating leukemia cutis is the control of systemic disease. In CLL, treatment consists of alkylating agents including chlorambucil and cyclophosphamide, associated with purine analogs (e.g. fludarabine). When associated with the latter, Rituximab has recently shown high disease response. ${ }^{3}$

Dermatologists must be aware of the diversity of cutaneous lesions in patients with leukemia. Besides the risk of bacterial and fungal infections, BLC can also simulate facial cellulitis in CLL patients.]

\title{
REFERENCES
}

1. Su WP, Buechner SA, Li CY. Clinicopathologic correlations in leukemia cutis. J Am Acad Dermatol. 1984;11:121-8.

2. Kikuchi N, Yamamoto T. Bullous leukemia cutis. Eur J Dermatol. 2012;22:148-9.

3. Batycka-Baran A, Baran W, Dzietczenia J, Mazur G. Effective treatment of leukemia cutis with combination of rituximab, cladribine, and cyclophosphamide in patient with B-cell chronic lymphocytic leukemia. Ann Hematol. 2011;90:979-80.

4. Plaza JA, Comfere NI, Gibson LE, Colgan M, Davis DM, Pittelkow MR, et al. Unusual cutaneous manifestations of B-cell chronic lymphocytic leukemia. J Am Acad Dermatol. 2009;60:772-80.

5. Kang YS, Kim HS, Park HJ, Lee JY, Kim HO, Cho BK, et al. Clinical characteristics of 75 patients with leukemia cutis. J Korean Med Sci. 2013;28:614-9.

6. Gabriela L, Peryassú D. Dermatologic iconography. Leukemia cutis. An Bras Dermatol. 1966;41:181-3.

\author{
MAILING ADDRESS: \\ Hélio Amante Miot \\ Departamento de Dermatologia e Radioterapia da FMB- \\ Unesp, SN. \\ Campus universitário \\ 18618-000 - Botucatu - SP \\ Brazil \\ E-mail: heliomiot@fmb.unesp.br
}

How to cite this article: Caldato LS, Britto JS, Niero-Melo L, Miot HA. Bullous leukemia cutis mimicking facial cellulitis. An Bras Dermatol. 2016:91(2):248-9. 